\title{
Editorial
}

\section{Marketing científico: investigadores y prosumidores en la ciencia.}

\section{Edwin Andrés Sepúlveda Cardona*}

Forma de citar este artículo en APA:

Sepúlveda Cardona, E. A. (2021). Marketing científico: investigadores y prosumidores en la ciencia [Editorial]. Poiésis, (41), 14-18. https://doi.org/10.21501/16920945.4188

Los eslabones para la difusión de la ciencia crecen y se diversifican al compás propio de las evoluciones tecnológicas digitales. El vértigo de los datos, la celeridad de la información, el crecimiento exponencial de plataformas (Martel, 2014) y la inclusión de planes publicitarios y de marketing en las actividades académicas y científicas, catalizan estrategias y tácticas que son comparables con las de otras industrias comerciales (Fenner, 2012).

Decía Van Noorden (2014) -en un artículo de la revista Nature con 787 citas en Google Scholar- que Twitter, Facebook, Linkedln (las generales) y Researchgate, Academia y Mendeley (las científicas) son las redes sociales de mayor impacto para encontrar pares científicos, publicar contenidos, compartir links, discutir activamente de investigaciones, descubrir nuevos papers recomendados, contactar con visitantes habituales y ser interrogados, en detalle, sobre sus proyectos y producción. En otras palabras, una eclosión de actividades hiperconectadas, una transtextualidad de contenidos y un mainstream de acciones oportunas para la visibilización, el impacto y la transferencia de conocimiento en sociedades red.

De igual modo, estamos asistiendo a una ebullición y expansión de canales digitales y plataformas disruptivas que propician oportunidades dialógicas especializadas y refinadas, porque debemos recordar que el medio es el mensaje (Strate, 2012). La digitalización y la transformación tecnológica -empujada en parte por la 
virtualización en pandemia- ha permitido una maduración de medios, un auge de herramientas y una oportunidad para proyectos, soluciones y marcas emergentes que son core en los procesos de visibilización de contenidos y productos científicos.

La lista puede ser infinita: libros en Amazon, shortlinks cuantificables de Bit.ly, cuentas en CrossRef, Delicious, ePrints, Figshare, Github, Goodreads, PLOS, Reddit, SlideShare, entre otras decenas de canales -alternativos- que hacen parte de un tejido hipermediático y son fuente cienciométrica para PlumX, para la Red Knowmetrics o para Semantic Scholar (Li, Peng \& Du, 2021), por ejemplo. Y que permiten la extracción de datos y de información de alto valor que impacta en la apropiación social del conocimiento, en citas, descargas e índices $\mathrm{H}$, lo que, en resumen, es un marketing de la ciencia para atraer a nuevos públicos.

Este funne/ de atracción evidencia una arquitectura tangible de un marketing científico ecléctico que emula del marketing convencional. Incluso, un marketing científico transmediático, que reúne múltiples formatos y que conforman un ecosistema digital medible y cuantificable (SepúlvedaCardona \& Suárez Quiceno, 2020): publicaciones en blogs, capítulos, casos, conferencias, infografías de conjuntos de datos, entrevistas, cartas, boletines de prensa, posters, reportes, presentaciones, videos y hasta tráilers de publicaciones científicas (Jauregui Caballero \& Ortega Ponce, 2020). Simultáneamente, este abanico digital de formatos, canales, herramientas, apps, software e instrumentos han permitido desarrollar KPI (Key Performance Indicator) especializados para el marketing científico que se planea, ejecuta y mide, tal cual, como una campaña publicitaria tradicional de una agencia de marketing (Sepúlveda-Cardona, 2020). Empresas, marcas, programas y profesionales son capaces de medir hasta cinco categorías de KPI distintas para cada investigador y científico:

- Usage: clicks, vistas, descargas, reproducciones de video...

- Captures: marcadores, favoritos, códigos embebidos, observadores...

- Mentions: publicaciones en blogs, comentarios, revisiones, links...

- Social media: favoritos, likes, compartidos, guardados, posts...

- Citations: en Scopus, PubMed, Patentes, Semantic y Google Scholar.

En consecuencia, el aparato científico de los países se ha movilizado -tal vez tarde en los países de América Latina- hacia planes y estrategias innovadoras como prácticas de un mercadeo científico que adopta tendencias de industrias comerciales. En el caso de Colombia, el Ministerio de la Ciencia, Tecnología e Innovación ha permitido una serie de estrategias novedosas en sus políticas y directrices que son visibles desde sus plataformas para investigadores y grupos de investigación.

En el año 2021 ha generado en su plataforma de CvLac -que es la hoja de vida científica de los investigadores colombianos- una nueva categoría denominada Producción de estrategias y contenidos transmedia: "son producciones convergentes de contenidos en distintos soportes, digitales o físicos, que involucran distintos productos comunicativos y circulan a través de diferentes plataformas aprovechando su naturaleza, ofreciendo una experiencia unificada y coordinada". (Minciencias, 2021, p. 100) 
Estas experiencias transmedia hacen parte de los productos de divulgación pública de la ciencia donde se puede registrar los medios del producto, público objetivo, género literario, rutas de circulación, enfoques diferenciales, conceptualización del formato, componente transmediático, lineamientos conceptuales, referentes creativos, características técnicas y estructura narrativa, entre otros. Todos ellos son, además, obligatorios para la validación ante el sistema nacional de ciencia.

Algo semejante sucede en la categoría de Desarrollo web (Minciencias, 2021, p. 105), también como estrategia de apropiación social del conocimiento y divulgación pública de la ciencia donde los investigadores deben registrar una información similar: público objetivo, componente digital, conceptualización del formato, género, UX o experiencia del usuario, wireframes y mockups, lineamientos conceptuales, referentes creativos, características técnicas y tecnológicas, y estructura narrativa. Como se puede observar, estas nuevas labores científicas, que son tensiones para muchos rezagados, pero un hábito para científicos con competencias digitales, son clave en la visibilización del conocimiento e integran un mashup de tecnologías y habilidades de marketing para la ciencia.

Finalmente, los investigadores y los científicos se enfrentan a múltiples y nuevos desafíos dadas las fuertes competencias al interior y el exterior de las comunidades y a las restricciones financieras y económicas que afectan el impacto de los proyectos. Estamos en un punto de inflexión sobre los roles de los científicos que, al parecer, tienden hacia actividades propias de un prosumidor científico. Los prosumidores (Scolari, 2018), ustedes y nosotros, científicos o no, estamos necesitando de múltiples sistemas, experiencias, canales y contenidos para visibilizar nuestra producción (textual, hipertextual, visual, audiovisual) en los ecosistemas digitales. Contenidos científicos, análisis, metanálisis, investigaciones, reflexiones, revisiones sistematizadas o sistemáticas, etc., que necesitan de dos tipos de narrativas: una, para los humanos y, otra, para los robots digitales (spiders); que permita que ese contenido, además de ser visible, sea perdurable y reutilizable (Borgman et al., 2012). Estamos hablando de contenidos con una narrativa de fácil lectura para científicos, para la apropiación social, para legos, pero, también, de una semántica para las máquinas digitales.

En este orden de ideas, los prosumidores científicos están realizando contenidos digitales hacia los Frontend de las revistas, de los repositorios y de las plataformas que buscan una usabilidad y lecturabilidad para las sensibilidades humanas. $Y$ del mismo modo, construyen, diseñan, escriben un backend estratégicos para Google y todo su ecosistema, para la matainformación de los journal, para otros metabuscadores generales y científicos. Tareas vertiginosas, bucles infinitos de un espectro digital largo y ancho. 


\section{Conflicto de intereses}

El autor declara la inexistencia de conflicto de interés con institución o asociación comercial de cualquier índole.

\section{Referencias}

Borgman, C. L., Wallis, J. C., \& Mayernik, M. S. (2012). Who's Got the Data? Interdependencies in Science and Technology Collaborations. Computer Supported Cooperative Work (CSCW), 21(6), 485-523. https://doi.org/10.1007/s10606-012-9169-z

Fenner, M. (2012). One-click science marketing. Nature materials, 11(4), 261-263. https://www. nature.com/articles/nmat3283

Jauregui Caballero, A., \& Ortega Ponce, C. (2020). Narrativas transmediáticas en la apropiación social del conocimiento. Revista Latina de Comunicación Social, (77), 357-372. https://doi. org/10.4185/RLCS-2020-1462

Li, X., Peng, S., \& Du, J. (2021). Towards medical knowmetrics: representing and computing medical knowledge using semantic predications as the knowledge unit and the uncertainty as the knowledge context. Scientometrics, 1-27. https://doi.org/10.1007/s11192-021-03880-8

Martel, F. (2014). Smart: Internet(s), la investigación. Taurus.

Minciencias. (2021). Manual de usuario CvLac [Online]. https://minciencias.gov.co/sites/default/ files/ckeditor_files/D103M06\%20Manual\%20de\%20Usuario\%20CVLAC\%20V01do.pdf

Scolari, C. A. (2018): Las leyes de la interfaz: Diseño, ecología, evolución, tecnología. Gedisa.

Sepúlveda-Cardona, E.A. (2020). Agencias de publicidad, e-branding y ecosistemas digitales. Análisis articulado de los metamedios de las agencias de publicidad de Argentina, Chile, Colombia, España, Estados Unidos, México y Perú. [Tesis de doctorado, Universidad Autónoma de Barcelona]. TDX. Tesis doctorales en Xarxa.

Sepúlveda-Cardona, E., \& Suárez Quiceno, C. (2020). Ecosistemas digitales para la formación: Redes sociales, ebooks y ciencia 2.0. En: C. Suárez Quiceno (Comp.), Piedra, papel y pixel: el texto digital en juego (pp. 51-64). Fondo Editorial Universidad Católica Luis Amigó. https:// www.funlam.edu.co/uploads/fondoeditorial/551_Piedra,_papel_y_pixel.pdf 
Strate, L. (2012). El medio y el mensaje de McLuhan: La tecnología, extensión y amputación del ser humano. Infoamérica: Iberoamerican Communication Review, (7), 61-80.

Van Noorden, R. (2014). Scientists and the social network. Nature news, 512(7513), 126. https:// www.nature.com/news/online-collaboration-scientists-and-the-social-network-1.15711 